Stud. Univ. Babeş-Bolyai Math. 65(2020), No. 1, 77-91

DOI: $10.24193 /$ subbmath.2020.1.07

\title{
The critical point of a sigmoidal curve
}

\author{
Ayse Humeyra Bilge and Yunus Ozdemir
}

\begin{abstract}
Let $y(t)$ be a monotone increasing curve with $\lim _{t \rightarrow \pm \infty} y^{(n)}(t)=0$ for all $n$ and let $t_{n}$ be the location of the global extremum of the $n$th derivative $y^{(n)}(t)$. Under certain assumptions on the Fourier and Hilbert transforms of $y(t)$, we prove that the sequence $\left\{t_{n}\right\}$ is convergent. This implies in particular a preferred choice of the origin of the time axis and an intrinsic definition of the even and odd components of a sigmoidal function. In the context of phase transitions, the limit point has the interpretation of the critical point of the transition as discussed in previous work [3].
\end{abstract}

Mathematics Subject Classification (2010): 34A99.

Keywords: Sigmoidal curve, critical point, Fourier transform, Hilbert transform.

\section{Introduction}

A sigmoidal function $y(t)$ is a monotone increasing function with horizontal asymptotes as $t \rightarrow \pm \infty$. Such functions occur in probability theory and in a variety of applications that represent the passage between two stable states, in particular in phase transitions.

In previous work [5] we have modeled the sol-gel transition of the polyacrylamidesodium alginate composite in terms of the Susceptible-Infected-Removed (SIR) epidemic model that represents the spread of an epidemic in a closed society. This model was shown to be in good agreement with the aforementioned gelation phenomena and we tried to take advantage of an exact mathematical model to search for the exact instant of onset of the sol-gel transition $[3,4]$. We computed higher derivatives of the sigmoidal curve representing the phase transition, up to orders 20 to 30 and we observed that the points where their reach their absolute extrema seemed to have a limit point, as shown in Fig. 1.

This point agreed qualitatively with the gel point of the polyacrylamide-sodium alginate composite and we proposed to define the "critical point of a sigmoidal curve" 
$y(t)$ as the limit of the sequence of points where the higher derivatives $y^{(n)}(t)$ reach their absolute extreme values [3].
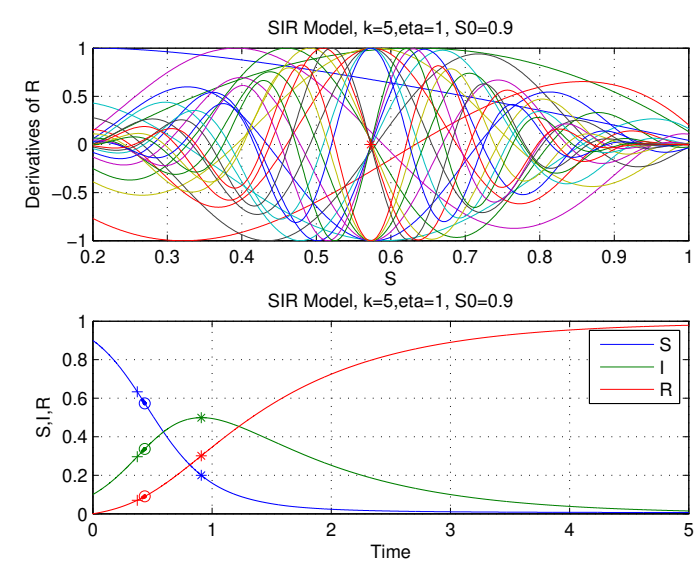

Figure 1. The Susceptible-Infected-Removed dynamical system $S^{\prime}=-k S I, I^{\prime}=k S I-\eta I, R^{\prime}=\eta I$, as a model for the gelation phenomena. (a) The first 24 derivatives of $R(t)$ normalized to 1 for $k=5, \eta=1$ and $S_{0}=0.9$ are plotted against $S(t)$, which is a monotone decreasing function of $t$. The phase transition point is indicated by (*). (b) The time domain plots of the solution curves $S, I, R$. The phase transition point $t_{c}$ indicated by $(o)$ is located between the maximum of $I, t_{m}$ denoted by $(*)$ and the inflection point of $I, t_{a}$ denoted by $(+)$. The derivatives of the sigmoidal function $R(t)$ are plotted versus $S(t)$, which is a monotone function of time.

Referring to Fig. 1(a), we first note that there seems to be a gap in the zero set of the derivatives; that is, the normalized absolute values of the odd derivatives agglomerate quickly near the point shown by $(*)$, while the absolute extrema of the even derivatives approach this point much more slowly. On the other hand, the work of Polya [9] on the zeros of the set of derivatives of an analytic function applied to a smooth sigmoidal curve implies that there should be no gap in the set of zeros. Due to the computational limitations of the SIR system, we worked with the logistic growth function and we could in fact see that the gap closes when derivatives up to order 200 are included, as shown in Fig. 2. Nevertheless, despite the strong evidence for the existence of a critical point, we were unable to prove even the simplest observed fact that the absolute extreme values of the odd derivatives of the logistic growth curve are located at $t=0$.

The aim of the present work is the study of the existence and the location of the "critical point of a sigmoidal curve" as described Section 2, Definition 1. In earlier stages of this study, the existence of a critical point was thought to be a peculiarity of the SIR system, but later on after working with numerous examples we came up 


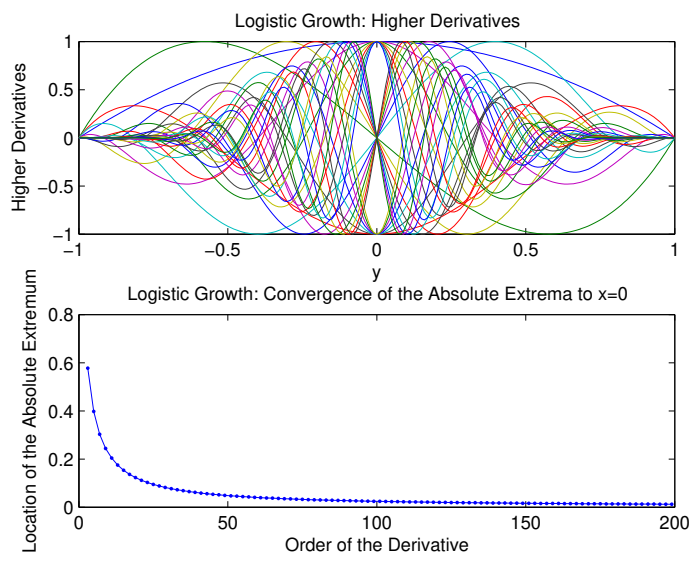

Figure 2. The normalized derivatives of the logistic growth $y^{\prime}=$ $\left(1-y^{2}\right)($ a) The plot of derivatives up to order 30 with respect to $y$. All odd derivatives reach their absolute extremum at the origin but there seems to be a gap in the zero set. (b) The distance of the first zero of the $n$th derivative to the origin. The apparent gap seems to close after $n=200$.

with the belief that it is a consequence of the general properties of sigmoidal curves. Intuitively, the critical point of an odd sigmoidal curve is expected to be $t=0$, but there was no guess on where the critical point of curves with no symmetry would be located.

The main results of the paper are presented in Section 3. In Section 3.1, we prove the existence of critical point for the general case (Proposition 2), then in Section 3.2, we consider odd sigmoidal curves and use milder assumptions to prove that $t=0$ is the critical point. For the case with no symmetry, the location of the critical point is crucially related to an appropriate choice of the origin of the time axis, in such a way that the phase of the Fourier transform of the first derivative is asymptotically constant. This leads to an intrinsic choice of origin, hence an intrinsic definition of the even and odd components, provided that the relevant assumptions are satisfied. With this choice of origin, $t=0$ turns out to be the critical point, provided that it exists.

The plan of the paper is as follows. The definitions and theorems necessary for subsequent derivations are presented in Section 2. In Section 3.1, we first prove the existence of the critical point for the general case, then, in Section 3.2, we give alternative proofs for sigmoidal curves with symmetry, using weaker assumptions. Basic properties of the Fourier and Hilbert transforms are given in the Appendix. 


\section{Preliminaries}

In Section 2.1, we illustrate the existence and non-existence of critical points for certain sigmoidal curves. We define the critical point of a sigmoidal curve and present our basic assumptions in Section 2.2. In Section 2.2, we define the intrinsically even and odd components and in Section 2.3, we prove certain results related to the properties of the envelope.

\subsection{The existence and non-existence of the critical point}

We will denote the sigmoidal curve as $y(t)$ and its first derivative that is a localized hump by $f(t)$, hoping that there will be no confusion when we refer to even and odd derivatives of $y$ or $f$. Let $t_{n}$ be the point where the $n$th derivative $y^{(n)}(t)$ reaches its extreme value and let $y_{n}=y^{(n)}\left(t_{n}\right)$. Based on our observations we expect that the subsequences $\left\{t_{2 k}\right\}$ and $\left\{t_{2 k+1}\right\}$ converge at different rates. For example, in the case of an odd sigmoidal curve, $t_{2 k+1}=0$ for each $k$, while $\left\{t_{2 k}\right\}$ converges slowly as seen from Fig. 2(b).

The standard and generalized logistic growth curves provide examples to the existence of the critical point for symmetrical and asymmetrical growth. The standard logistic growth curve is the sigmoidal curve $y(t)=\tanh (t)$, while the generalized logistic growth curve with horizontal asymptotes at -1 and 1 is given by $y(t)=$ $-1+2\left[1+k e^{-\beta t}\right]^{-1 / \nu}$, where $k>0, \beta>0$ and $\nu>0$. The parameter $k$ can be adjusted by a time shift, $\beta$ corresponds to a scaling of time and $\nu$ is the key parameter that determines the shape of the growth. For $k=1$ the critical point is located at $t=0$ (see Example 2). In Fig. 3(a), all even derivatives of the sigmoidal function are zero at $t=0$; the apparent gap is still discernable despite a much higher number of derivatives are plotted. The behavior of the generalized logistic growth (Fig. 3(b)) is more or less the same except that the zeros of even derivatives are not fixed but they agglomerate near $t=0$.

The Gompertz function with the same asymptotes is given by $y(t)=-1+$ $2 \exp \left(-e^{-\beta t}\right)$. This function can be expressed as the limit of the generalized logistic family for $k=1 / n, \nu=1 / n$, as $n \rightarrow \infty$ and provides an example to the non-existence of the critical point. As seen in Fig. 3(c), the normalized derivatives do not accumulate and the critical point seems to have moved to negative infinity. The derivatives of the Gompertz function of orders 20, 30 and 40 are presented in Fig. 4, in order to display the shift of the wave packets towards minus infinity.

The standard logistic growth curve occurs as the solution of the "SusceptibleInfected-Susceptible" (SIS) model. In a study of the formation of (reversible) physical gels, [6], we showed that that generalized logistic growth curves are solutions to a modified form of the (SIS) model and we used the results of Example 2, in Section 3, to determine the gel point directly, using the approximation of experimental results by generalized logistic growth curves, known also as "5-point sigmoids".

In [2] we expressed sufficient conditions for the existence of a critical point of a sigmoidal curve in terms of the Fourier transform of the first derivative. For the sigmoidal curves that arise as solutions of the SIR model, we could only give numerical evidence for the existence of the critical point. But for the solutions of the SIS 
model expressed in terms of generalized logistic growth functions, we could express the location of the critical point in terms of the parameters of the generalized logistic growth curve [6] where we used without proof the expression of the Fourier transform of its first derivative.

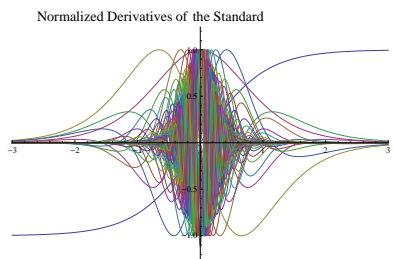

(a)

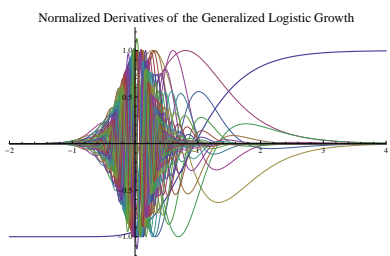

(b)

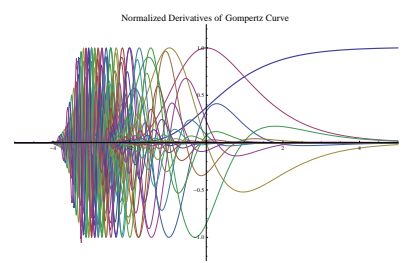

(c)

Figure 3. (a) Normalized derivatives of the standard logistic growth; (b) Normalized derivatives of the generalized logistic growth $(\beta=1, k=1, \nu=1 / 5)$ up to order 30. The apparent gap is still discernable in both figures, despite the large number of derivatives plotted. The behavior of the generalized logistic is more or less the same except that the zeros of even derivatives are not fixed. (c) Normalized derivatives of the Gompertz function. The Gompertz function is the limit of the generalized logistic family, the critical point seems to have moved to negative infinity.

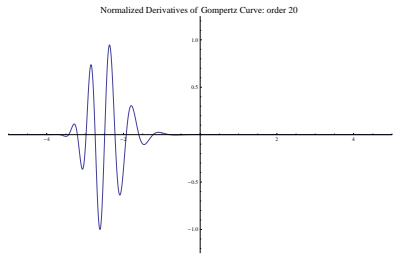

(a)

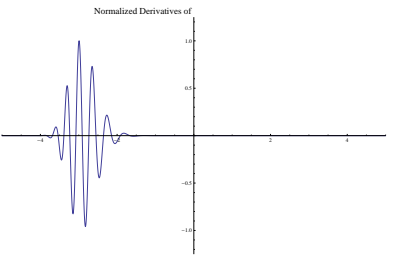

(b)

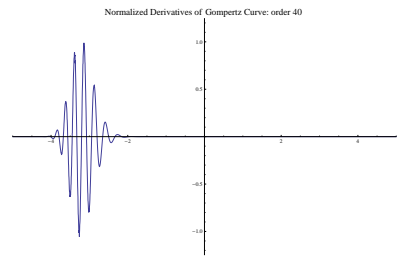

(c)

Figure 4 . The time domain plots of the 20th, 30th and 40th derivatives of the Gompertz function respectively in (a), (b) and (c). The wave packets are shifted to left towards minus infinity.

\subsection{Basic definitions}

A sigmoidal function $y(t)$ is a monotone increasing function with horizontal asymptotes $y_{1}$ and $y_{2}$ as $t \rightarrow \pm \infty$ and with $\lim _{t \rightarrow \pm \infty} y^{(n)}(t)=0$ for all $n \geq 1$. We propose the following definition for the "critical point".

Definition 2.1. Let $y(t)$ be a sigmoidal curve and assume that the set of points where the even derivatives and the odd derivatives reach their absolute extremum converge to the same point. The common limit of these derivatives, if it exists, is called the critical point of the sigmoidal curve. 
The location of the critical point brings into consideration an intrinsic definition of being even or odd, hence a preferred choice of origin of the time axis. If $f(t)$ is even, then its Fourier transform $F(\omega)$ is real and even, but if $f(t)$ is shifted in time, then $F(\omega)$ is no longer real, but its phase is linear. The other way around we can recognize an intrinsically even function by looking to the phase of its Fourier transform. From this point of view, the preferred origin for the time axis is given by the shift that will make $F(\omega)$ real.

In the general case where $f(t)$ has no symmetry, $F(\omega)$ has a nonzero phase. But if the phase has an oblique asymptote $\alpha \omega+\phi_{0}$, then after a time shift, it will be asymptotically constant. Thus, the preferred origin of time is obtained by the time shift that makes the phase of $F(t)$ asymptotically constant, provided that the phase has an oblique asymptote.

We use this property to define the intrinsically even and intrinsically odd functions via their Fourier transform.

Definition 2.2. Let $f(t)$ be a function whose Fourier transform $F(\omega)$ exists. $f(t)$ is called intrinsically even if there is real number $\alpha$ such that $e^{-i \alpha \omega} F(\omega)$ is real. $f(t)$ is called intrinsically odd if there is real number $\alpha$ such that $e^{-i \alpha \omega} F(\omega)$ is pure imaginary.

If there is no real number $\alpha$ such that $f(t-\alpha)$ is neither even or nor odd, then we define its intrinsically even and odd components provided that the phase of $F(\omega)$ is asymptotically linear, i.e, $F(\omega)=|F(\omega)| e^{i \phi(\omega)}$ where $\phi(\omega)$ has an oblique asymptote with slope $\alpha$, as $\omega \rightarrow \pm \infty$.

Definition 2.3. Let $f(t)$ be a function such that the Fourier transform $F(\omega)$ exists and the phase of $F(\omega)$ has an oblique asymptote with slope $\alpha$ as $\omega \rightarrow \pm \infty$. Then, the intrinsically even and odd components of $f(t)$ are the inverse Fourier transforms of the real and imaginary parts of $e^{-i \alpha \omega} F(\omega)$.

\subsection{The envelope of the derivatives}

Let $f(t)$ be a derivative of a sigmoidal function. We will prove that the magnitude of the analytic representation $f_{A}(t)$ (as defined in the Appendix) gives the envelope of $f(t)$ in the sense that $f(t)$ touches $\left|f_{A}(t)\right|$ between any two consecutive zeros.

Proposition 2.4. Let $f(t)$ be a real function whose Fourier and Hilbert transforms exit. If $t_{1}$ and $t_{2}$ are any two consecutive zeros of $f(t)$, then there is a $t_{3}$ such that $t_{1}<t_{3}<t_{2}$ and $f\left(t_{3}\right)= \pm\left|f_{A}(t)\right|$.

Proof. Writing $f_{A}(t)=A(t) e^{i \varphi(t)}$, we can express $f(t)$ and $f_{h}(t)$ as

$$
f(t)=\left|f_{A}(t)\right| \cos (\varphi(t)), \quad f_{h}(t)=A(t) \sin (\varphi(t)) .
$$

If $t_{1}$ and $t_{2}$ are two consecutive zeros of $f(t)$, then we should have $\varphi\left(t_{1}\right)=\frac{\pi}{2}+k \pi$ and $\varphi\left(t_{2}\right)=\frac{\pi}{2}+(k+1) \pi$. Thus, provided that $\varphi(t)$ is continuous, there will be a time $t_{3}, t_{1}<t_{3}<t_{2}$, such that $\varphi\left(t_{3}\right)=(k+1) \pi$, hence, $f_{h}\left(t_{3}\right)=0$. Thus the zeros of $f(t)$ and $f_{h}(t)$ alternate. It follows that $f\left(t_{3}\right)= \pm\left|f_{A}\left(t_{3}\right)\right|$ for some $t_{1}<t_{3}<t_{2}$. The local extremum of $f(t)$ in between $t_{1}$ and $t_{2}$ is denoted by $t_{4}$. If $f(t)$ is positive (negative) on $\left(t_{1}, t_{2}\right)$ and $f^{\prime}\left(t_{3}\right)$ is negative (positive), then $t_{4}<t_{3}$, while if $f(t)$ is positive (negative) on $\left(t_{1}, t_{2}\right)$ but $f^{\prime}\left(t_{3}\right)$ is positive (negative), then $t_{3}<t_{4}$. 


\section{The existence and the location of the critical point of a sigmoidal curve}

For the case with symmetry, i.e, for an odd sigmoidal curve $y(t)$, the Fourier transform of the first derivative, $F(\omega)$ is real. All odd derivatives of $y(t)$ have a local maximum at $t=0$. We expect the local maximum to be located at $t=0$. For a sigmoidal curve with no symmetry, we will show that the location of the critical point is given by the linear phase factor of $F(\omega)$.

The motivation for the choice of the assumptions in Proposition 2 is based on the following observation. In the examples studied, higher derivatives of the sigmoidal curve look like wave packets, hence in the frequency domain, the Fourier transform of the derivatives should have a nearly band bass spectrum. If the Fourier transform of all derivatives have the same (constant) phase, then in the time domain, the wave packets are centered at the same point. As this condition is not satisfied for sigmoidal curves without symmetry, the best that we can expect is that all derivatives have asymptotically a constant phase (possibly after a shift of time) and the band pass spectrum moves to infinity as the order of differentiation increases.

Before proceeding to the proofs, we start by examples to illustrate the behavior of curves with no symmetry.

Example 3.1. If $f(t)$ is an even localized hump, then its even derivatives are even and its odd derivatives are odd functions. By adding these with appropriate multiples one can generate positive pulses with no symmetry. As an example, the first derivative of the standard logistic growth, $\operatorname{sech}^{2}(t)$, is an even pulse. We obtain a positive pulse with no symmetry by adding a multiple of its second derivative

$$
f(t)=y^{(1)}(t)=\operatorname{sech}^{2}(t)-\lambda\left[-2 \operatorname{sech}^{2}(t) \tanh (t)\right] \quad(0<\lambda<0.5),
$$

the peak being located at the right of the point $t=0$. The Fourier transform of $f(t)$ is

$$
F(\omega)=(1-i \lambda \omega) \sqrt{\frac{2}{\pi}} \frac{\pi \omega / 2}{\sinh (\pi \omega / 2)} .
$$

The phase of $F(\omega)$ is $\phi(\omega)=\arctan (\operatorname{Im}(F) / \operatorname{Re}(F))=\arctan (-\lambda \omega)$. As $\omega \rightarrow \pm \infty$, $\phi(\omega)$ approaches $\mp \pi / 2$, hence $F(\omega)$ has asymptotically constant phase.

Example 3.2. The first derivative of the generalized logistic growth is

$$
f(t)=\frac{2 k \beta}{\nu} e^{-\beta t}\left(1+k e^{-\beta t}\right)^{-1-1 / \nu} .
$$

The Fourier transform of $f(t)$ is [1]

$$
F(\omega)=\sqrt{\frac{2}{\pi}} k^{-\frac{i \omega}{\beta}} \frac{\Gamma\left(1+\frac{i \omega}{\beta}\right) \Gamma\left(\frac{1}{\nu}-\frac{i w}{\beta}\right)}{\Gamma\left(\frac{1}{\nu}\right)} .
$$

Since $k^{-\frac{i \omega}{\beta}}=e^{-i\left(\frac{\ln k}{\beta}\right) \omega}$, it follows for $k \neq 1$, there is a linear phase factor in $F(\omega)$. Let $k=1$ and $\beta=1$. For $1 / \nu=n$, one can use the property $\Gamma(\bar{z})=\overline{\Gamma(z)}$ repeatedly to see that

$$
F(\omega)=\frac{1}{\Gamma(n)}(1-i \omega)(2-i \omega) \ldots(n-1-i \omega) F_{s}(\omega)
$$



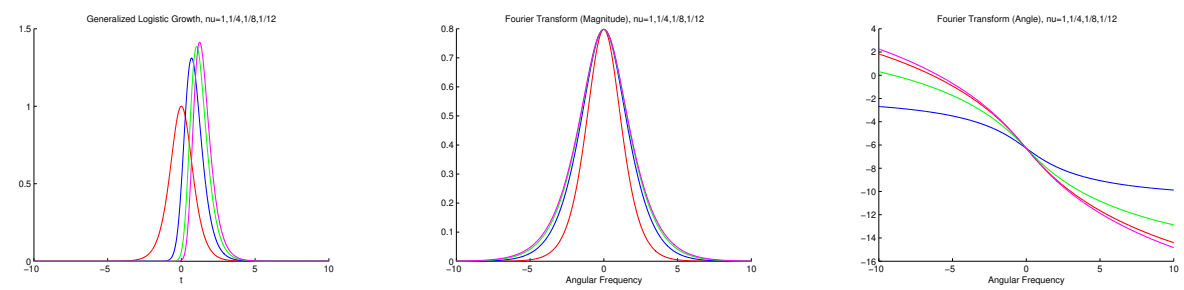

Figure 5. a) Time domain plots of the first derivative of the generalized logistic growth for $1 / \nu=1,1 / 4,1 / 8,1 / 12$. b) The magnitude of the Fourier transform, c) The angle of the Fourier transform.

where $F_{s}(\omega)$ is the Fourier transform of the standard logistic growth. The multiplicative factor is a complex polynomial; in particular the zeros of its real part are bounded, hence as $\omega \rightarrow \pm \infty$, the phase goes to a multiple of $\pi / 2$. It follows that, for each $n$, the phase is asymptotically constant (provided that $k=1$ ). The time domain plot, the magnitude and the phase of the Fourier transforms of the generalized logistic family for $\nu=1,1 / 4,1 / 8$ and $1 / 12$ are presented in Fig. 5. We note that as $1 / \nu$ increases, the phase approaches to its horizontal asymptotes more and more slowly.

\subsection{Existence of the critical point: The general case}

We will now prove that if $f(t)$ satisfies asymptotically constant phase and bandpass hypotheses, to be specified below, then the critical point is located at $t=0$. Note that the asymptotically constant phase condition is trivially satisfied when $f(t)$ is even.

Proposition 3.3. Let $f(t)$ be the first derivative of a sigmoidal curve $y(t)$ and $f^{(n)}(t)$ be its nth derivative. If

(i) the Fourier transform of $f(t)$ has the form $F(\omega)=|F(\omega)| e^{-i \alpha \omega} e^{i \psi(\omega)}$ where $\alpha$ is a constant and $\psi(\omega)$ has horizontal asymptotes,

(ii) for $\omega>0, \omega^{n}|F(\omega)|$ has a single maximum at $\omega_{n}$ and the $\omega_{n}$ 's are unbounded,

(iii) the spectrum is localized in the sense that there are constants $\omega_{a}$ and $\omega_{b}$ (depending on $n$ ), such that

$$
\lim _{n \rightarrow \infty} \int_{|\omega|<\omega_{a}} \omega^{n}|F(\omega)| d \omega=\lim _{n \rightarrow \infty} \int_{|\omega|>\omega_{b}} \omega^{n}|F(\omega)| d \omega=0,
$$

then the sigmoidal curve $y(t)$ has a critical point located at $t=\alpha$.

Proof. For simplicity assume that $\alpha=0$. If the Fourier transform of $f(t)$ is $F(\omega)$, then the Fourier transform of $f(t-\alpha)$ is $e^{-i \alpha \omega} F(\omega)$. We will express $\left|f^{(n)}(t)\right|$ using the Fourier inversion formula and compare it with $\left|f^{(n)}(0)\right|$. The assumption (iii) implies that for all $\varepsilon$, there is $N>0$ such that for $n>N$, there are constants $\omega_{a}$ and $\omega_{b}$ (depending on $n$ ), such that

$$
\int_{|\omega|<\omega_{a}} \omega^{n}|F(\omega)| d \omega<\frac{\varepsilon}{8} \text { and } \int_{|\omega|>\omega_{b}} \omega^{n}|F(\omega)| d \omega<\frac{\varepsilon}{8} .
$$


Thus for $n$ large, the contribution from low and high frequencies are negligible, hence the main contribution comes from a neighborhood of $\omega_{n}$. By assumption (ii) the $\omega_{n}$ 's are unbounded and using the asymptotically constant phase assumption we obtain the estimates below:

$$
\int_{\omega_{a} \leq|\omega| \leq \omega_{b}} \omega^{n}|F(\omega)|\left(1-e^{i \psi(\omega)}\right) d \omega<\frac{\varepsilon}{2}
$$

Letting $I$ to be the set defined by $\omega_{a} \leq|\omega| \leq \omega_{b}$, we obtain upper bounds for $f^{(n)}(t)$ as below:

$$
\begin{aligned}
\sqrt{2 \pi}\left|f^{(n)}(t)\right| & =\left|\int_{-\infty}^{\infty} \omega^{n} F(\omega) e^{i \omega t} d \omega\right| \leq \int_{-\infty}^{\infty} \omega^{n}|F(\omega)| d \omega \\
& =\int_{|\omega|<\omega_{a}} \omega^{n}|F(\omega)| d \omega+\int_{|\omega|>\omega_{b}} \omega^{n}|F(\omega)| d \omega+\int_{\omega \in I} \omega^{n}|F(\omega)| d \omega \\
& =\frac{\varepsilon}{4}+\int_{\omega \in I} \omega^{n}|F(\omega)| d \omega
\end{aligned}
$$

We estimate the integral above as

$$
\begin{aligned}
\int_{\omega \in I} \omega^{n}|F(\omega)| d \omega & =\left|\int_{\omega \in I} \omega^{n}\right| F(\omega)|d \omega| \\
& =\left|\int_{\omega \in I} \omega^{n}\right| F(\omega)\left|\left(1-e^{i \psi(\omega)}\right) d \omega+\int_{\omega \in I} \omega^{n}\right| F(\omega)\left|e^{i \psi(\omega)} d \omega\right| \\
& \leq\left|\int_{\omega \in I} \omega^{n}\right| F(\omega)\left|\left(1-e^{i \psi(\omega)}\right) d \omega\right|+\left|\int_{\omega \in I} \omega^{n}\right| F(\omega)\left|e^{i \psi(\omega)} d \omega\right| \\
& \leq \frac{\varepsilon}{2}+\left|\int_{\omega \in I} \omega^{n}\right| F(\omega)\left|e^{i \phi_{0}} e^{i \psi(\omega)} d \omega\right| \\
& \leq \frac{\varepsilon}{2}+\left|\int_{\omega \in I} \omega^{n} F(\omega) d \omega\right| .
\end{aligned}
$$

Finally we estimate the last term as

$$
\begin{aligned}
\left|\int_{\omega \in I} \omega^{n} F(\omega) d \omega\right| & =\left|\int_{-\infty}^{\infty} \omega^{n} F(\omega) d \omega-\int_{|\omega|<\omega_{a}} \omega^{n} F(\omega) d \omega-\int_{|\omega|>\omega_{b}} \omega^{n} F(\omega) d \omega\right| \\
& \leq\left|\int_{-\infty}^{\infty} \omega^{n} F(\omega) d \omega\right|+\left|\int_{|\omega|<\omega_{a}} \omega^{n} F(\omega) d \omega\right|+\left|\int_{|\omega|>\omega_{b}} \omega^{n} F(\omega) d \omega\right| \\
& \leq\left|\int_{-\infty}^{\infty} \omega^{n} F(\omega) d \omega\right|+\int_{|\omega|<\omega_{a}} \omega^{n}|F(\omega)| d \omega+\int_{|\omega|>\omega_{b}} \omega^{n}|F(\omega)| d \omega \\
& \leq \frac{\varepsilon}{4}+\sqrt{2 \pi}\left|f^{(n)}(0)\right|
\end{aligned}
$$

It follows that $f^{(n)}(t) \leq f^{(n)}(0)+\varepsilon$ hence as $t=0$ is the critical point.

The sequence $\omega_{n}$ can be obtained by maximizing $\omega_{n}|F(\omega)|$, i.e, by equating its first derivative to zero. We present in Fig. 6, a graphical display of the corresponding equality $\omega / n=F(\omega) / F^{\prime}(\omega)$ for the standard logistic growth. The comparison of the 


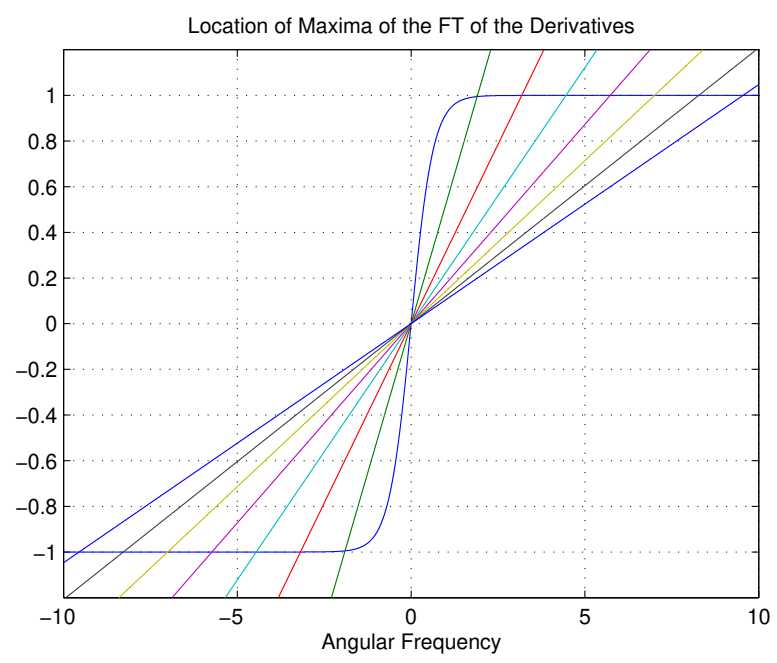

Figure 6. Graphical solution of the equation $\omega / n=F(\omega) / F^{\prime}(\omega)$ for the standard logistic growth.
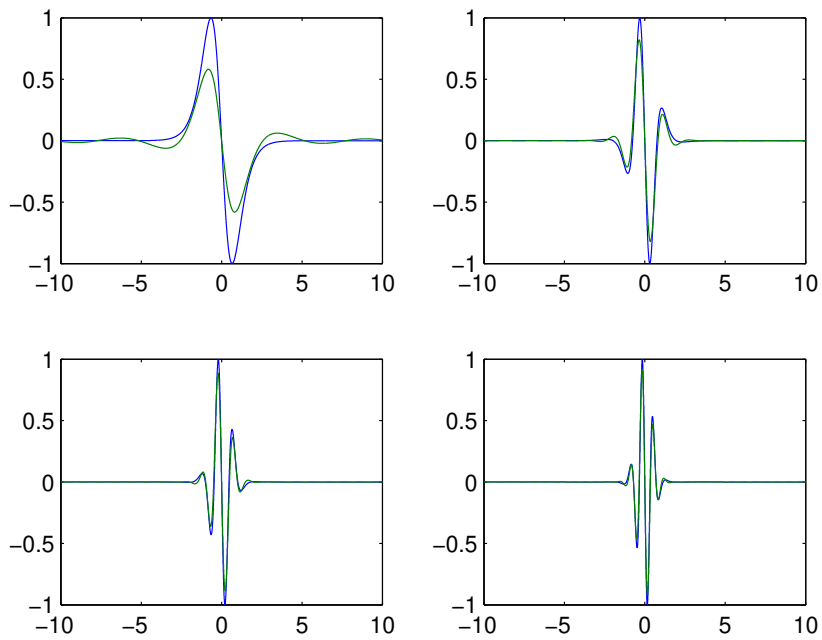

Figure 7. Comparison of the derivatives $f^{(n)}(t)$ of the standard logistic function with sinusoids of frequency $\omega_{n}$ modulating $\left|f_{A}^{(n)}(t)\right|$.

higher derivatives with sinusoids of frequency $\omega_{n}$ modulating the amplitude of the corresponding analytical representation are shown in Fig. 7. 


\subsection{The existence of the critical point: Odd sigmoidal curves}

In this section we will prove the existence of the critical point of an odd sigmoidal curve under a different set of assumptions. We first prove that the odd derivatives of an odd sigmoidal function $y(t)$ reach their global extreme values at $t=0$ (Proposition $3.4)$. Then we prove that the global extreme value of the even derivatives of $y(t)$ is the local extreme value that is closest to $t=0$ (Proposition 3.5) and the sequence of points where the even derivatives of $y(t)$ reach their global extreme values converge to $t=0$ (Corollary 3.6). The time domain plots and the magnitude of the Fourier and Hilbert transforms of the standard logistic growth are displayed in Fig. 8.
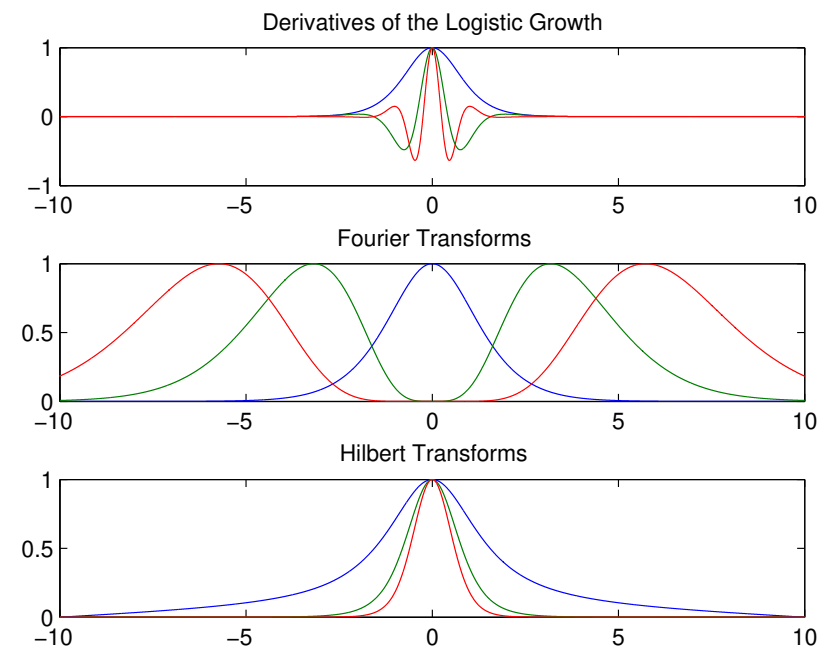

Figure 8. Normalized graphs of the time domain variations, the magnitude of the Fourier transform and the magnitude of the analytic representation for $y(t), y^{(4)}(t)$ and $y^{(8)}(t)$. As the order of differentiation increases, the time domain pulses get narrower while the frequency spectrum spreads out.

The simplest property that has to be proved is the fact that if $f(t)$ is an even, the local extremum at $t=0$ is the global one. We prove this by requiring the "monotonicity" of the envelope of $y^{(n)}(t)$, expressed in terms of its analytic representation.

Proposition 3.4. Let $f(t)$ be a real, even function and assume that that the magnitude of its analytical representation $\left|f_{A}(t)\right|$ has a single local maximum. Then

$$
|f(t)| \leq|f(0)| \text {. }
$$

Proof. Recall that $f_{A}(t)=f(t)+i f_{h}(t)$, where $f_{h}(t)$ is the Hilbert transform of $f(t)$. Since $f_{A}^{2}(t)=f^{2}(t)+f_{h}^{2}(t),|f(t)| \leq\left|f_{A}(t)\right|$. When $f(t)$ is even, $f_{h}(t)$ is odd, hence, $f(0)=f_{A}(0)$. Since $\left|f_{A}(t)\right|$ is monotone decreasing, $|f(t)| \leq\left|f_{A}(0)\right|=|f(0)|$. 
We next prove that the global extremum of the even derivatives of an odd sigmoidal curve is the one closest to $t=0$.

Proposition 3.5. Let $f(t)$ be a real, odd function such that the magnitude of its analytical representation $\left|f_{A}(t)\right|$ has a single local maximum. Then $|f(t)| \leq\left|f\left(t_{0}\right)\right|$ where $t_{0}$ is the location of the first local extremum of $f(t)$ for $t>0$.

Proof. Since $f(t)$ is odd, $f(0)=0$. For simplicity assume that $f^{\prime}(0)>0$. Let $t_{1}$ be the first zero of $f(t)$ for $t>0$. By the alternation of roots, there is a point $t_{2}<t_{1}$ at which $f\left(t_{2}\right)=\left|f_{A}\left(t_{2}\right)\right|$ and since $\left|f_{A}(t)\right|$ is decreasing, $f(t)$ is reaching its first local maximum at some $t_{0}<t_{2}$. It follows that $|f(t)|<\left|f\left(t_{0}\right)\right|$.

Finally we prove that the global extreme values of the derivatives converge to $t=0$, i.e, there is no gap between the maximum of $\left|y_{A}^{(2 k)}(t)\right|$ and the global extremum of $y^{(2 k)}(t)$.

Corollary 3.6. Let $y(t)$ be an odd sigmoidal function and assume that for each $n$, $\left|y_{A}^{(n)}(t)\right|$ has a single local maximum. Then $t=0$ is the limit point of the global extreme values of $y^{(2 k)}(t)$.

Proof. For $n=2 k+1$, Proposition 3.4 implies that the global extremum is at $t=0$. For $n=2 k$, we will prove that the global extremum of $y^{(2 k+2)}$ occurs earlier than the global extremum of $y^{(2 k)}$. By Proposition 3.5 above, the global extremum is the first local extremum. Let $y^{(2 k)}(0)=0$, assume that $y^{(2 k+1)}(0)>0$ and let $t_{1}$ be the first intersection of $y^{(2 k)}$ with its envelope, $y^{(2 k)}\left(t_{1}\right)=\left|y_{A}^{(2 k)}\left(t_{1}\right)\right|$. The global extreme value of $y^{(2 k)}$ is at some $t_{2}<t_{1}$, since the envelope is decreasing. Then, $y^{(2 k+1)}\left(t_{2}\right)=0$, $y_{h}^{(2 k+1)}\left(t_{2}\right)$ is tangent to the envelope, hence it has its global extremum at $t_{3}<t_{2}$. Finally, $y_{h}^{(2 k+2)}\left(t_{3}\right)=0$, hence $y^{(2 k+2)}(t)$ is tangent to the envelope at this point and it has its global extremum at some $t_{4}<t_{3}$. Hence the global extreme values form a decreasing sequence that converge to $t=0$.

Typical examples of the even and odd derivatives of the standard logistic growth and their envelopes are presented in Fig. 9, as to illustrate how the proofs work.

For the case with no symmetry, we again need to assume that $\left|y_{A}^{(n)}(t)\right|$ has a single maximum, but the location of this maximum, that we denote as $t_{n, *}$ changes with $n$. The comparison of $\left|y_{A}^{(n)}(t)\right|$ for sigmoidal curves with and without symmetry is given in Fig. 10.

\section{Appendix A. The Fourier and Hilbert transforms}

The Fourier transform: The Fourier transform of a function $f(t), \mathcal{F}(f)=F(\omega)$ is defined as

$$
F(\omega)=\frac{1}{\sqrt{2 \pi}} \int_{-\infty}^{\infty} f(t) e^{-i \omega t} d t,
$$




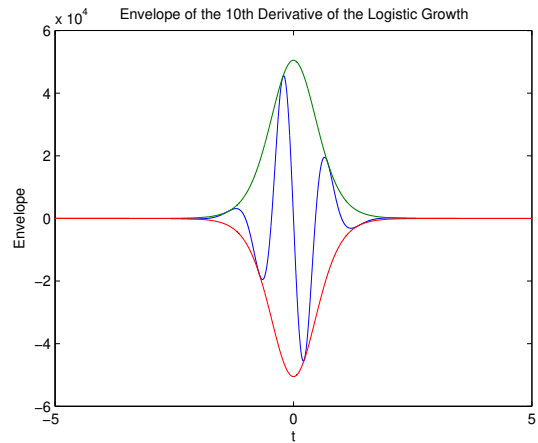

(a)

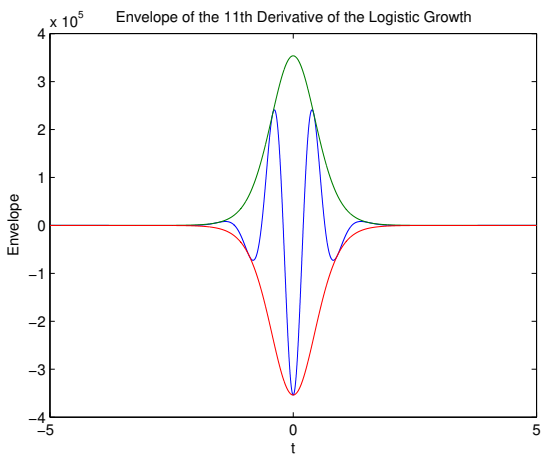

(b)

Figure 9. The envelope of the 10th (a) and of 11th (b) derivative of the standard logistic growth function.

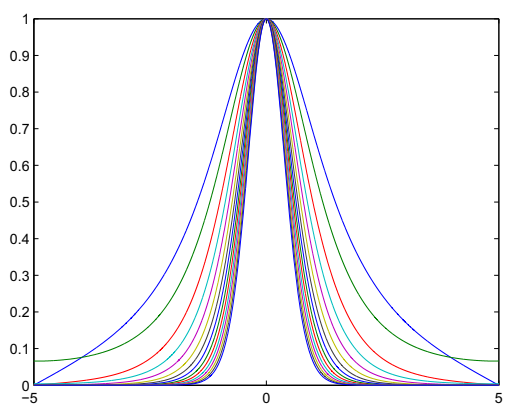

(a)

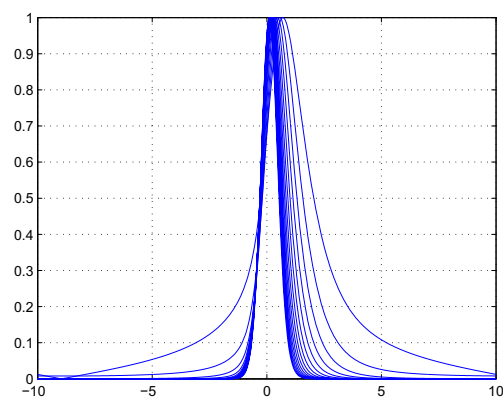

(b)

FiguRE 10. Envelopes of normalized derivatives up to order 15: The standard logistic growth function (a) and generalized logistic growth function with $\beta=2, k=1, \nu=1 / 5$ (b).

provided that the integral exists in the sense of Cauchy principal value [8]. If $f(t)$ is in $L^{1}$, then its Fourier transform exists. Since a sigmoidal function is finite as $t \rightarrow \infty$, its first derivative is in $L^{1}$. We can recover $f(t)$ from the inverse transform by

$$
f(t)=\frac{1}{\sqrt{2 \pi}} \int_{-\infty}^{\infty} F(\omega) e^{i \omega t} d \omega
$$

The Fourier transform of the odd sigmoidal function exists in the sense of Cauchy principal value.

The Hilbert transform and the analytic representation: For our purposes, the simplest description of the Hilbert transform is given by its relation to the Fourier transform 
[7]. Given $f(t)$ and its Fourier transform $F(\omega)$, we define the function $F_{A}(\omega)$ by

$$
F_{A}(\omega)=\left\{\begin{array}{cc}
2 F(\omega) & , \quad \omega>0 \\
0 & , \quad \omega<0
\end{array} .\right.
$$

The inverse transform is a complex function that is called the "analytic representation" $f_{A}(t)$ of $f(t)$. The imaginary part of $f_{A}(t)$ is the Hilbert transform $f_{h}(t)$ of $f(t)$.

$$
f_{A}(t)=f(t)+i f_{h}(t)=\sqrt{\frac{2}{\pi}} \int_{0}^{\infty} F(\omega) e^{i \omega t} d \omega .
$$

The existence of the Hilbert transform necessitates that $f(t)$ be in $L^{p}$ for $1<p<\infty$; for $L^{1}$ functions, it exists in $L^{1, \text { weak }}$ ([10], Lemma V.2.8).

Symmetry properties: The property, $\mathcal{F}(\overline{f(t)})=\overline{F(-\omega)}$ implies that if $f(t)$ is real then $F(-\omega)=\overline{F(\omega)}$. Thus if $f(t)$ is real, $F(\omega)$ will be real provided that $F(-\omega)=$ $F(\omega)$. The scaling property $f(a t) \rightarrow \frac{1}{|a|} F\left(\frac{\omega}{a}\right)$ implies that $f(-t) \rightarrow F(-\omega)$, hence if $f(t)$ is real and even, then $F(\omega)$ is real and even. Similarly, if $f(t)$ is real and odd, then $F(\omega)$ is pure imaginary and odd. We note that if $f(t)$ is even (odd), its Hilbert transform is odd (even).

Differentiation: The effect of differentiation in the time domain is multiplication by $i \omega$ in the frequency domain. Thus

$$
f^{(n)}(t) \rightarrow(i \omega)^{n} F(\omega)
$$

Convolution and modulation: There is a correspondence between products and convolutions in the time and frequency domains; multiplication in the time domain leads to convolution in the frequency domain, i.e,

$$
f(t) g(t) \rightarrow \frac{1}{\sqrt{2 \pi}} F(\omega) * G(\omega) .
$$

The "modulation" of a low frequency signal in the time domain is the multiplication of this signal by a sinusoidal function of fixed (usually high) angular frequency $\omega_{0}$. In the frequency domain, the Fourier transform of the low frequency function is convolved with the Fourier transform of the sinusoid. The Fourier transform of a pure sinusoid is not defined in the usual sense, but it is represented as the Dirac $\delta$ functions occurring at $\pm \omega_{0}$ and convolution carries the spectrum of the low frequency signal to the frequencies $\pm \omega_{0}$. Since the Fourier transform of a complex exponential is a $\delta$-function, we have the correspondence below:

$$
f(t) e^{i \omega_{0} t} \rightarrow F\left(\omega-\omega_{0}\right)
$$

Time shift: As an analogue of multiplication with a complex exponential in the time domain, the multiplication of a function in the frequency domain by a linear phase factor leads to a shift in the time domain:

$$
e^{-i \alpha \omega} F(\omega) \rightarrow f(t-\alpha)
$$




\section{References}

[1] Bilge, A.H., Ozdemir, Y., The Fourier transform of the first derivative of the generalized logistic growth curve, Int. J. Adv. Eng. Pure Sci., 32(2020), no. 1, 51-56.

DOI: $10.7240 /$ jeps.598861

[2] Bilge, A.H., Ozdemir, Y., Determining the critical point of a sigmoidal curve via its Fourier transform, Edited by Vagenas, E.C. and Vlachos, D.S., 5th International Conference on Mathematical Modeling in Physical Sciences (IC-MSQUARE 2016) May 2326, 2016, Athens, GREECE, J. Phys. Conf. Ser., 738(2016).

DOI:10.1088/1742-6596/738/1/012062.

[3] Bilge, A.H., Pekcan, O., A mathematical description of the critical point in phase transitions, Internat. J. Modern Phys. C, 24(2013).

[4] Bilge, A.H., Pekcan, O., A mathematical characterization of the gel point in sol-gel transition, Edited by: Vagenas, E.C., Vlachos, D.S., Bastos, C. et al., 3rd International Conference on Mathematical Modeling in Physical Sciences (IC-MSQUARE 2014) August 28-31, 2014, Madrid, SPAIN, J. Phys. Conf. Ser., 574(2015), Article Number: 012005.

[5] Bilge, A.H., Pekcan, O., Gurol, V., Application of epidemic models to phase transitions, Phase Transitions, 85(2012), 1009-1017.

[6] Bilge, A.H., Pekcan, O., Kara, S., Ogrenci, S., Epidemic models for phase transitions: Application to a physical gel, 4th Polish-Lithuanian-Ukrainian Meeting on Ferroelectrics Physics Location: Palanga, LITHUANIA, 5-9 September 2016, Phase Transitions, 90(2017), no. 9, 905-913.

[7] Duoandikoetxea, J., Fourier analysis, Graduate Studies in Mathematics, 29, AMS, Providence, RI, 2001.

[8] Papoulis, A., The Fourier Integral and its Applications, McGraw-Hill Co., New York, 1962.

[9] Polya, G., On the zeros of the derivatives of a function and its analytic character, Bull. Amer. Math. Soc., 49(1942), 178-191.

[10] Stein, E.M., Weiss, G.L., Introduction to Fourier Analysis on Euclidean Spaces, Princeton University Press, Princeton, 1971.

Ayse Humeyra Bilge

Kadir Has University,

Faculty of Engineering and Natural Sciences,

34083 Istanbul, Turkey

e-mail: ayse.bilge@khas.edu.tr

Yunus Ozdemir

Eskişehir Technical University,

Department of Mathematics,

26470 Eskisehir, Turkey

e-mail: yunuso@eskisehir.edu.tr 